\title{
A look at hadronization via high multiplicity
}

\author{
E. Kokoulina ${ }^{* 1,2}$, A. Kutov ${ }^{3}$, V. Nikitin ${ }^{1}$, V. Riadovikov ${ }^{4}$, and A. Vorobiev ${ }^{4}$ \\ ${ }^{1}$ JINR, Joliot-Curie 6, Dubna, Moscow region, 141980, Russian Federation, \\ ${ }^{2}$ GSTU, Gomel, Belarus, \\ ${ }^{3}$ Institute of Physics and Mathematics Komi SC UrD RAS, Kommunisticheskaja st., 24, \\ Syktyvkar,167000, Russian Federation \\ ${ }^{4}$ IHEP, Science sq. 1, Protvino, Moscow region, 142281, Russian Federation \\ E-mail: kokoulina@jinr.ru, kutovedm.komisc.ru, nikitin@jinr.ru, \\ vorobieveihep.ru, riadovikoveihep.ru
}

Multiparticle production is studied experimentally and theoretically in QCD that describes interactions in the language of quarks and gluons. In the experiment the real hadrons are registered. For transfer from quarks and gluons to observed hadrons various phenomenological models are used. In order to describe the high multiplicity region, we have developed a gluon dominance model (GDM). It represents a convolution of two stages. First stage is described as a part of QCD. For second one (hadronisation), the phenomenological model is used. To describe hadronisation, a scheme has been proposed, consistent with experimental data in the region of its dominance. Comparison of this model with data on e+e- annihilation over a wide energy interval (up to 200 $\mathrm{GeV}$ ) confirms the fragmentation mechanism of hadronisation, the development of the quarkgluon cascade with energy increase and domination of bremsstrahlung gluons. The description of topological cross sections in pp collisions within of GDM testifies that in hadron collisions the mechanism of hadronisation is being replaced by the recombination one. At that point, gluons play an active role in the multiparticle production process, and valence quarks are passive. They stay in the leading particles, and only the gluon splitting is responsible for the region of high multiplicity. GDM with inclusion of intermediate quark charged topologies describes topological cross sections in $p \bar{p}$ annihilation and explains initial linear growth in the region of negative values of a secondary correlative momentum vs average pion multiplicity with increasing of energy. The scaled variance of a neutral pion number measured by us is rising abruptly in the region of high total multiplicity and differs from Monte Carlo predictions by seven standard deviations. The growth of fluctuations of the neutral pion number in this region may indicate the formation of a pion (Bose-Einstein) condensate. While searching for this collective phenomenon, events with a predominance of a large number of neutrals $\left(n_{0}=16\right)$ among total multiplicity $\left(n_{t o t}=32\right)$ have been found. Despite the growth of fluctuations on the neutral number, their average remains equal to $1 / 3$ of the total pion number. The proposed hadronization scheme is capable of describing the most basic processes of multiparticle production.

XIII Quark Confinement and the Hadron Spectrum - Confinement2018

31 July - 6 August 2018

Maynooth University, Ireland

${ }^{*}$ Speaker. 


\section{Introduction}

Development of high energy physics has been considerably accelerated after the appearance of the strong interaction theory or quantum chromodynamics (QCD) [1]. It can be pointed out that two experimental stages influenced its development. The first stage is connected with experiments at hadron accelerators, and the second stage is the appearance of electron-positron beams. These experimental results require the explanation, especially important for description of multiparticle production. Such physicists like Fermi, Pomeranchuk, Hagedorn, Dremin, Brodsky and others developed phenomenological schemes based on statistical description. The bootstrap model of Hagedorn predicts the existence of extreme temperature. This behaviour is now interpreted as the formation of quark-gluon plasma.

Using QCD elementary processes, Konishi, Ukava and Veneciano [2] and A. Giovannini [3] built a system of stochastic equations to calculate multiplicity distributions (MD) of partons in quark and gluon jets at high energy collisions. Taking into account the two main elementary processes (gluon splitting and bremsstrahlung) gives MD of quarks and gluons in these jets. MD of partons in a quark jet is a well-known negative binomial distribution (NBD), and a Yule-Furry distribution describes MD in a gluon jet..

\section{2. $e^{+} e^{-}$annihilation and three-gluon decay of bottomonium}

For comparison with the experimental data, these distributions cannot be used because free quarks are not observed experimentally, due to confinement which has been accepted without proofs so far. To eliminate this difficulty, at the description of MD for $e^{+} e^{-}$-annihilation to hadrons, a hypothesis of local parton-hadron duality (LoPHD) has been proposed, according to which the hadronization of quarks and gluons occurs softly, without significant momentum transfer between partons. Thus, for description of MD in that process, two stages are taken: the first stage or the quark-gluon $(q g)$ fission, to which the pQCD can be applied, and the hadronization stage, described phenomenologically. The LoPHD hypothesis was quite consistent with the experiment, while energy of accelerators was not so high to be developing enough $q g$-cascades. Experiments at the DESY accelerator confirmed formation of quark and gluon jets, which testified in favour of QCD.

In order to describe $\mathrm{MD}$ in $e^{+} e^{-}$annihilation to hadrons at energies from several tenths to hundreds $\mathrm{GeV}$, the two-stage model (later renamed the gluon dominance model, GDM) has been offered $[4,5,6,7]$. It's based on the description of MD of partons forming from $q \bar{q}$-pair at the first stage of that process by Giovannini's distributions [3]. We also added a phenomenological scheme at the second stage (hadronization of quarks and gluons). In this model, for hadrons from the parton (quark or gluon) jet at the hadronization stage, a binomial distribution (BD) with a negative second correlation moment $\left(f_{2}=\overline{n(n-1)}-\bar{n}^{2}, n\right.$ - multiplicity, $\bar{n}$ - its average value) is used. This choice is based on experimental data [8]. At energies of a few $\mathrm{GeV}$ when the number of partons at the stage of the $q g$-cascade is small, the hadronization is predominant and determines the sign of the second correlation moment. Its experimental value in this area is negative.

With increasing energy, it's obvious that the $q g$-ascade is developing. It becomes prevailing over the hadronization stage. In accordance with Giovannini's approach [3], this cascade is de- 
scribed by NBD with a positive second correlation moment and two parameters, an average gluon multiplicity and a $k_{p}$ parameter that has a sense of the inverse temperature $(\mathrm{T})$ of the $q g$-system, $k_{p} \sim T^{-1}$. Change of the second correlation moment sign with increasing energy is confirmed experimentally. This model is built by a convolution of two stages describing both NBD and BD. It describes the experimental MD of charged particles and indicates the active role of gluons in their formation. It is interesting to follow the change of its parameters with increasing energy at both stages.

In accordance with the two stage model, at the first stage, the average multiplicity of gluons $(\bar{m})$ increases with energy and can be described by a logarithmic dependence (Fig. 1, a). At the same time, the NBD parameter $k_{p}$ is decreasing, which indicates an increase in temperature of the $q g$ system. At the second stage, the parameters of the model $N_{p}$ and $\bar{n}_{p}^{h}$, where $p$ is $q$ or $g$, determine, correspondently, the maximum and the average number of hadrons produced from single quark or gluon in the region of hadronization where application of the perturbation theory of QCD is difficult. Comparison with experimental data shows the gluon jet is softer, and its parameter $\bar{n}_{p}^{h}$ remains almost constant and close to one in the energy range from 10 to $200 \mathrm{GeV}$ (Fig. 1, b).

Such behaviour confirms the LoPHD hypothesis and the fragmentation mechanism of hadronization [10]. At the fragmentation mechanism, the initial high energy quark emits a bremsstrahlung gluon which splits to $q \bar{q}$ pair. Then a pair's quark picks up a convenient quark (antiquark) from a vacuum and forms an observed meson. In this case, mesons turn out predominant particles. Experiments at RHIC show that the ratio of baryons to mesons are considerably less than one in the peripheral region. The creation of heavy quark pairs occurs but it's suppressed in comparison with the central region.

As an example, MD in $e^{+} e^{-}$-annihilation at $189 \mathrm{GeV}$ is shown in Fig. 2, a. As opposed to the predictions of numerous Monte-Carlo generators, GDM correctly describes the MD on the tail of a large multiplicity, and it also indicates the sources of the observed oscillations of the normalised correlation moments at high energy [6] namely developed $q g$-cascade hadronisation stage.

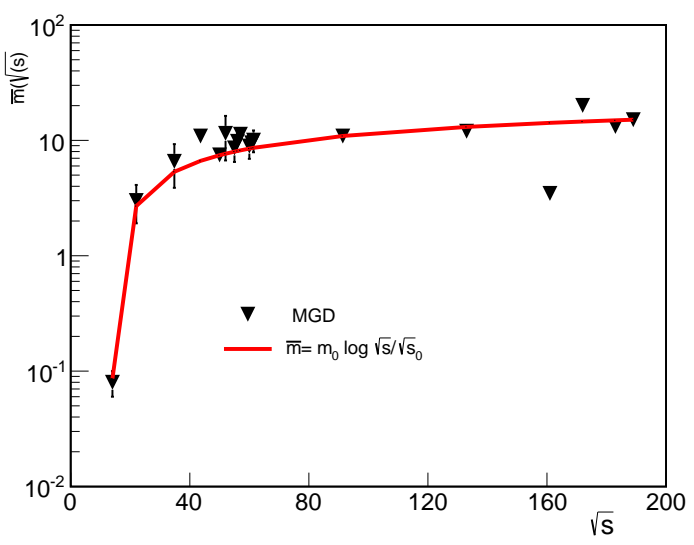

a

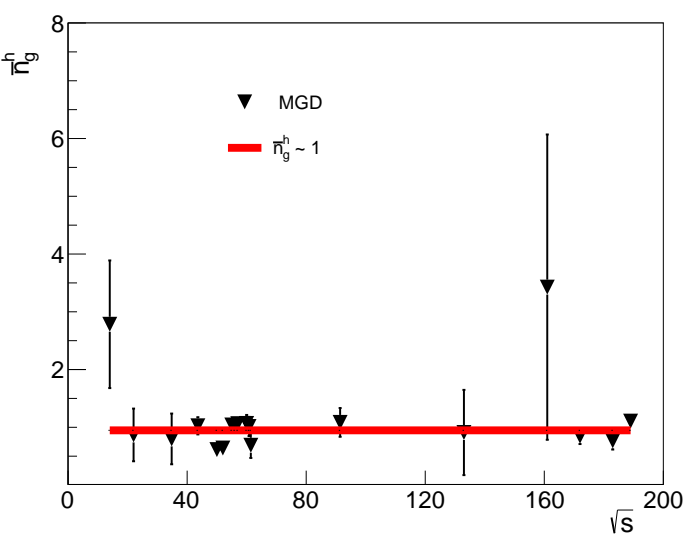

b

Figure 1: (a) The parameter $\bar{m}$, average multiplicity of gluons formed in the qg-cascade from a single gluon. (b) The hadronization parameter $\bar{n}_{g}^{h}$ ([4]) has sense of the average multiplicity of hadrons formed from single gluon The number of hadrons formed from one gluon during its passing of the second stage. Experimental data for the MD description of hadrons by GDM was taken from about $10 \mathrm{GeV}$ up to $200 \mathrm{GeV}$ [5] . 
The two-stage model, in particular, explains the jump between the average multiplicities in the three-gluon decay of heavy quarkonia $\Upsilon(9.4)$ and $\Upsilon(10.02)$ and in $e^{+} e^{-}$-annihilation at the same energy [5]. The oscillations of normalised cumulative moments can be described within this scheme [6].

The new stage, no less exciting in the study of nuclear matter, began with the appearance of high-energy hadron accelerators as well as heavy ion colliders with energies a few hundreds GeV. These studies are carried out at the large hadron collider (LHC) where particles are accelerated up to energy of several $\mathrm{TeV}$. The world society of physicists is discussing a future project of new generation accelerators with considerably higher energy (hundreds $\mathrm{TeV}$ ).

\section{3. $p p$ and $p \bar{p}$ interactions with high multiplicity}

The Thermalization project was advanced in 2004 at JINR (Dubna, Russia). Three institutes carried it out: JINR, IHEP (Protvino) and INP MSU (Moscow) at the SVD-2 setup (Spectrometer with a Vertex Detector) located on the extracted proton beam of the U-70 accelerator (IHEP). This project was aimed to search for collective phenomena in $p p$ interactions with a $50 \mathrm{GeV}$ proton beam in the region of multiplicity, which is several times larger than average value.

In this region of multiplicity, such phenomena as the formation of the pion condensate, an increased yield of soft photons, the formation of pion jets of the same sign and others are predicted. Before start of the experiment, a Monte-Carlo (MC) simulation was performed. A comparison of it with the data obtained by the Mirabelle collaboration at the U-70 accelerator showed that it significantly (about two orders of magnitude) underestimates the data on the tail of a large multiplicity $n_{c h}=18$. Therefore, we concluded that we should build an improved model consistent with the data in this area.

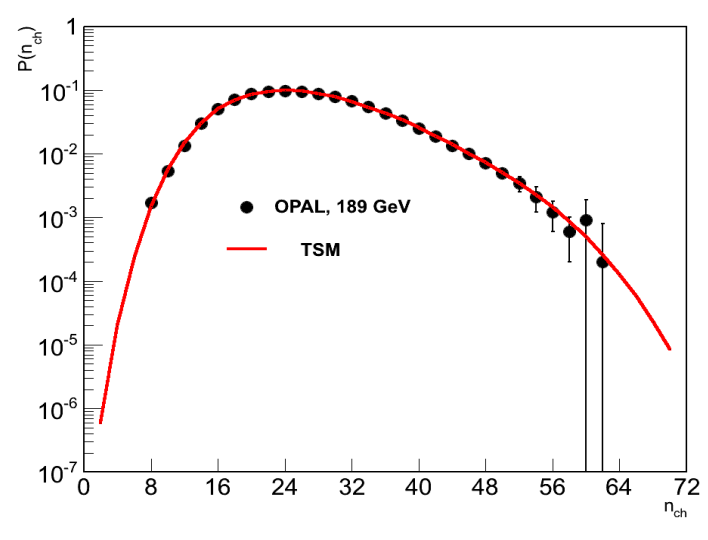

a

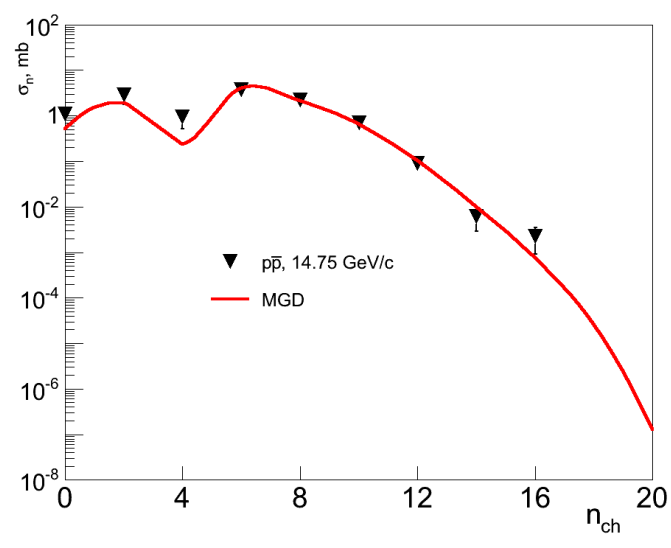

b

Figure 2: (a) MD of hadron in $e^{+} e^{-}$annihilation at $189 \mathrm{GeV}$ [6]. (b) Differences of topological cross sections between proton-antiproton and proton-proton $\left(\sigma_{n}=\sigma_{p \bar{p}}-\sigma_{p p}\right)$ interactions at the same energy about ten $\mathrm{GeV}[5]$.

We guessed what hadronization of quarks and gluons in hadron collisions occurs the same way as in $e^{+} e^{-}$annihilation. Just like in $e^{+} e^{-}$annihilation description, our modified GDM takes 
into account both of stages of multiparticle production. We analysed all available for us data on topological cross sections of hadronic interactions at energies up to several hundred $\mathrm{GeV}$ and found, the existing phenomenological models and Monte-Carlo codes did not predict behaviour in the region of high multiplicity. The Mirabelle Collaboration data had indicated to us the direction to develop our model.

We started modelling of a scheme of multiparticle production with the most common case when all valence quarks and a few, so-called, active gluons (appear at the moment of collision) are participants of $p p$ interactions. We use the term "active" gluon for those gluons that can give gluons of fission with the following quark pair formation. These pairs of quarks form observable hadrons (mesons and baryons) by combinatorial permutations. The hadronization stage of partons is described by the binomial law as well as in ${ }^{+} e^{-}$annihiltion.

In this case, parameters, which have the meaning of the average number of hadrons formed from quark or gluon, at the hadronization stage, $\bar{n}_{q(g)}^{h}$, accept values significantly less than the same parameters at the description of $e^{+} e^{-}$-annihilation. Therefore, we assumed that not all valence quarks are active, and we consistently reduced their number from three pairs to two, to one and, finally, all of them had been completely excluded and left in the leading particles. And then, parameter $\bar{n}_{g}^{h}$ began to grow and even slightly exceeded the value, corresponding to $e^{+} e^{-}$-annihilation.

Thus, the analysis of $p p$ interactions indicates the passivity of valence quarks and the active role of gluons in multiple production. Valence quarks remain in the leading nucleons, which are observed in the experiment. And only active gluons are the sources of secondary particles.

The model is implemented in two scenarios. The first scenario takes into account the division of gluons in $q g$-system at the first stage. Their appearance is described by the Poisson distribution and division by the Farry distribution. In the second scenario, the gluon splitting is not taken into account. The fragmentation of active gluons into hadrons is described in both scenarios the same way. It is assumed all stages of hadron interactions occur independently of each other.

Mirabelle and SVD-2 Collaborations measured topological cross sections of $p p$ interactions at $70 \mathrm{GeV}$. Their descriptions in both scenarios of GDM demonstrate the consistency of values of $\bar{n}_{g}^{h}$, the average multiplicity of hadrons produced from one gluon during its passing of the second stage. In the hadron collisions, it has small excess over $1: \bar{n}_{g}^{h}=1.63 \pm 0.12$. This is consistent with the recombination mechanism of hadronization taking place in a $q g$-medium, and not in vacuum as in the case of $e^{+} e^{-}$-annihilation.

The replacement of the hadronization mechanism at transition from the annihilation of leptons to the hadron and nuclei interactions is perfectly illustrated by B. Muller [10]. GDM indicates a logarithmic growth with the energy of $\bar{n}_{g}^{h}$, which at the top energy of the ISR accelerator, reaches the value $3.23 \pm 0.14$ [9].

Experiments at RHIC and LHC show growth of the ratio of baryon yield to the number of neutral pions. This ratio is approaching to 1 at the transition from peripheral to central collisions, which is also explained by the implementation of the recombination mechanism in central collisions. In $e^{+} e^{-}$-annihilation, this ratio is much less than 1 .

It should be noted in the first scenario, taking into account splitting of gluons, a fraction of active gluons is estimated as about $47 \%$ at $\mathrm{U}-70$ energy. The same estimation (about 50\%) has been obtained in QCD by A. H. Muller [11]. We assume that the remaining (not active, soft) gluons are picked up by the quarks formed by the splitting of active gluons. These quarks transform into 
observable hadrons. At the same time, excess of soft gluons can create an increased, in comparison with the existing models, yield of soft photons owing to the elementary process: $g+q \rightarrow \gamma+q$.

GDM indicates the predominance of splitting gluons in processes of multiple production at high energies. It explains discovered in heavy ion collisions at the RHIC setup, and then in proton collisions at LHC in high multiplicity events the long-radius correlations named ridges. We have shown [12] the formation of two gluons arising from a bremsstrahlung gluon by splitting at small angles to the initial direction of the valence quark and this splitting prevails in comparison with the serial emission by that quark of two gluons. In this case, we can observe a very narrow hadron jet with a wide variation of particles in rapidity.

Analysis of experimental data obtained at SVD-2 setup at interactions of 70-GeV proton beam with a hydrogen target and three nuclear targets $(\mathrm{C}, \mathrm{Si}, \mathrm{Pb})$ demonstrates the manifestation of a two-humped structure in a polar angle distribution in a region of large multiplicity. This was not observed in events with small multiplicity [7]. Such behaviour is often interpreted by analogy with Cherenkov radiation by collective emission of gluons by valence quarks, or, more often, by the formation of shock waves in a $q g$-medium. Comparison of our data with the formula of Cherenkov radiation permits us to estimate the refractive index of this medium. Its assessment is close to 1 which indicates the rarefaction of parton medium, in contrast to the medium formed in central collisions of relativistic heavy ions (the refractive index about 3 ).

GDM can describe differences between of topological cross sections for processes of protonantiproton annihilation and proton-proton interactions at $10 \mathrm{GeV}$. For that, we modify our scheme for the $p \bar{p}$ annihilation by the inclusion of the so-called intermediate charged quark topologies formed due the combination of valence (predominant) and sea quarks from colliding nucleons.

We can form the following combinations from quarks $(u, u, d)$ of the proton and antiquarks $(\bar{u}, \bar{u}, \bar{d})$ of the antiproton: "0"-, "2"-, "4"- and "6"- topologies. Neutral "0"-topology corresponds to the formation of three neutral pions from these partons , "2"-topology - the formation of two charged pions, $\pi^{+}$and $\pi^{-}$, and one neutral, $\pi^{0}$, by using only valence quarks. The remaining " 4 "and "6"-topologies form secondary pions not only from valence, but also from quarks of vacuum (sea quarks). Experiments indicate to the leading of two charged pions, which confirms this scheme [8].

In the modified GDM, we can neglect the rarest "6"-topology. The contribution of the other three combinations can be estimated from the comparison with the data. The parameters of hadronization are in agreement with the parameters determined from data of $p p$ interactions with minor deviations. The found relation between the topologies of "0" : "2" : "4" = $15: 40: 0.05$ indicates the main contribution is made by two main combinations of valence quarks ("0" and "2"). As shown in Fig. 2, b, GDM describes differences of topological cross sections between $p \bar{p}$ annihilation and $p p$ scattering at the same energy of colliding hadrons, including the appearance of two local maxima at the field of formation of two and six charged secondary particles. Moreover, "4"-topology is responsible for the appearance of the tail of high multiplicity.

At lower energies, the hadronization stage is predominant. In this case, calculated in GDM, the second correlation moment $f_{2}$ gives negative values and demonstrates the linear growth in the region of negative values with energy. In accordance with GDM, that behaviour is stipulated by increasing the average multiplicity of active gluons in a wide enough energy range in contrast to diffraction process like $p p$ collisions, in which valence quarks are staying in the leading nucleons. 
With growth of energy, the contribution of splitting gluons goes up which leads to a sign change of $f_{2}$ from negative to positive and to the broadening of MD.

\section{Pion (Bose-Einstein) condensate of neutral pions}

The next step we performed was the transition to study neutral pion production. We assumed the universality of hadronization in hadron interactions and used the available data from bubble chambers [13] on cross sections of $\pi^{0}$-mesons to describe MD of neutral pions by GDM. It turned out that such parameters of hadronization as the average value and the maximum possible number of $\pi^{0}$-mesons, which are formed at the stage of hadronization from one gluon source (active gluon), are comparable with the corresponding values for charged particles. Moreover, in GDM, the ratio between the average multiplicities of charged and neutral mesons is close to $2: 1$, which corresponds to the theoretical value.

Carrying out our experiment at U-70, we restored the multiplicity of neutral pions and investigated the region of high total multiplicity $n_{t o t}=n_{c h}+n_{0}$ as sum of charged and neutral particles. The SVD-2 setup allowed us to do that, as it includes an electromagnetic calorimeter. This calorimeter registers gamma-quanta (decay products of neutral pions). That detector is located on the beam line and behind the detectors that register charged particles.

The restoration of the total multiplicity allowed us to study such a collective phenomenon as the formation of pion or Bose-Einstein condensate (BEC). The theoretical substantiation of its observation has been predicted by the Begun and Gorenstein [14, 15]. They studied the fluctuations of the number of neutral pions at a fixed total pion multiplicity in the ideal pion gas statistical model and showed that the search for pion condensate have to be carried out by selecting events with high total multiplicity when the system is in thermal equilibrium but not chemical equilibrium.

At full equilibrium, the chemical potential of a pion system is equal to zero, and at the formation of the pion condensate, it's approaching to the pion mass. Such a state can be obtained by selecting events with a large number of pions, much more than the average multiplicity. The fluctuations of the number of neutral pions in this case are due to the dynamics of multiple production. The sharp growth of a scaled variance $\left(\omega=D / \bar{n}_{0}=\left(\bar{n}_{0}^{2}-\bar{n}_{0}^{2}\right) / \bar{n}_{0}\right)$ of the neutral pion number with an increase in the total pion multiplicity would be a signal for the BEC formation in proton collisions.

We found there isn't any growth of the scaled variance calculated for the simulated events in the whole simulation region. The reconstruction of $\pi^{0}$-mesons is based on using the simulation results. Event by event method is impossible in our case. Therefore, we worked out a new method for restoration of the number of events with a certain multiplicity of neutral mesons. About one million events were processed and the number of events with given multiplicity of neutral mesons have been obtained. Actually, rare events with high total multiplicity are observed with the number of $\pi^{0}$ mesons comparable to the multiplicity of charged particles: the events with $n_{t o t}=32$ pions is observed with the number of charged particles $n_{c h}=16$.

Our data are evidence of an increase of the scaled variance in the region of high total multiplicity, which reaches 7 standard deviations from simulated events at $n_{t o t} \sim 30$ [16]. The smallness of systematic errors in comparison with the statistical errors can be justified by the agreement of our data with the data of Mirabella's bubble chamber. 
Our theoretical estimations based on the formulas of statistical quantum physics indicate that in events with the maximum multiplicity, the born pions are in the BEC state. The Gorenstain's estimation of temperature for the pion condensate formation in $p p$ interactions are much bigger than temperature for condensation of bosonic atoms. It should be noted that SVD-2 data indicate that in events with the highest total multiplicity $\left(n_{\text {tot }}=36\right)$ neutral pions can be bigger than charged ones. Their number fluctuates strongly with an increase of $n_{t o t}$.

Thereby, study of high multiplicity events clarifies the hadronization mechanism, both charged and neutral hadrons, in different processes. We can describe MD in $e^{+} e^{-}$and $p \bar{p}$ annihilation, $p p$ interactions and three-gluon decay bottomonium $\Upsilon$ introducing the hadronization scheme in a wide energy region.

\section{References}

[1] F. Halzen and A.D. Martin. Quarks and Leptons. Acad. press, NY (1979).

[2] K. Konishi, A. Ukawa, and G. Veneziano. Jet calculus: A simple algorithm for resolving QCD jets. Nucl. Phys. B 157 (1979) 45-107.

[3] A. Giovannini, QCD jets as Markov branching processes. Nucl.Phys. B 61 (1979) 429-448.

[4] E.S. Kokoulina. Analysis of multiparticle dynamics in $e^{+} e^{-}$- annihilation into hadrons by two-stage model. XXXII ISMD, Alushta, Ukraine. 2002. World Scientific. 340-343.

[5] E.S. Kokoulina. High multiplicity study and gluon dominance model. Phys.Part.Nucl.Lett. 13 (2016) 74-78.

[6] E.S. Kokoulina, A.Ya. Kutov, and L.F. Babichev. Gluon dominance model and high multiplicity study Nonlin. Phenom. Complex Syst. 10 (2007) 291-296.

[7] E. Kokoulina, A. Kutov, and V. Nikitin Gluon Dominance Model and Cluster Production. Braz. J. Phys. 37 (2007) 785-787.

[8] J.G. Rushbrooke and B.R. Webber. High energy antiparticle-particle reaction differences and annihilations Phys. Rep. 44 (1978) 1-92.

[9] E.S. Kokoulina. Gluon dominance model. AIP. Conf. Proc. 828 (2006) 81-86.

[10] B. Muller. Hadronic signals of deconfinement at RHIC. Contribution to RBRC Scientific Articles. Proc. Series "New Discoveries at RHIC" Nucl. Phys. A 750 (2005) 84-97.

[11] A.H. Mueller. QCD in nuclear collisions Nucl. Phys. A 715 (2003) 20-33.

[12] E.A. Kuraev, S. Bakmaev, and E.S. Kokoulina, Azimuthal correlation of gluon jets created in $p p$, $p \bar{p}$ and $e^{+} e^{-}$collisions, Nucl. Phys. B851 (2011) 551-560.

[13] V.V. Ammosov et al. Average charged particle multiplicity and topological cross sections in 5-GeV/c and 69-GeV/c pp interactions Phys. Lett. B42 (1972) 519-521.

[14] V.V. Begun and M.I. Gorenstein. Bose-Einstein condensation of pions in high multiplicity events Phys. Lett. B 653 (2007) 190-195.

[15] V.V. Begun and M.I. Gorenstein. Bose-Einstein condensation in the relativistic pion gas: Thermodynamic limit and finite size effects. Phys. Rev. C 77 (2008) 064903.

[16] E. Kokoulina. Neutral Pion Fluctuations in pp Collisions at $50 \mathrm{GeV}$ by SVD-2. Progress of Theoretical Physics. Supplement. 193 (2012) 306-309. 